\title{
The right nutrition for the nutrition related diseases
}

\section{Roberto Vettor ${ }^{1}$}

Published online: 10 August 2020

(C) Springer Science+Business Media, LLC, part of Springer Nature 2020

\section{"Let food be your medicine and medicine be your food". Hippocrates of Kos (460-370 BC)}

To dedicate a special issue to the pathophysiological and clinical bases of a therapeutic strategy such as diet could seem unattractive for the current inflated proposals of colorful diets that are found in every magazine, newspaper or scientific journal, but now more than ever there is a need to bring diet therapy back to the consistency of scientific research and clinical methodology. These forward aims to trace the cultural motivations that led to this project.

The true founder of ancient medicine must be considered Hippocrates of Kos (460-370 BC), an itinerant professional who practiced in various places. His Hippocratic Corpus became the most essential medical document of all time even if it was probably a collaborative effort between various physicians of ancient Greece. "Let food be your medicine and medicine be your food". These are the famous and enlightening words of Hippocrates, who is also the author of the famous oath still used today by medical graduates; oath that commits the future doctor to act for the good of the patient, in full respect of his/her person. The father of medicine, well 400 years before the birth of Christ, had already understood the importance of nutrition in relation to our health and our psycho-physical well-being. "We are what we eat" Is it the man who makes the diet or is the diet that makes the man?Apparently it is the diet that makes man because humanity is born when it invents its diet, that is, it chooses what, how much, and how to eat.

Nowadays the world is full of different national and regional cuisines and thousands of different dishes. Despite the complexity of the food and its organoleptic qualities and the mixture of the various components with various colours and the context in which the food is consumed are all elements of

Roberto Vettor

roberto.vettor@unipd.it

1 Department of Medicine, Endocrine-Metabolic Unit, Padova University, Padova, Italy great importance, for practical reasons we could analyse and reduce to the basic molecules: carbohydrates, lipids and proteins. Each class of molecule is very important for the way our body works. So, what to choose? The nutritional content of what we eat determines the composition of our cell membranes, the growth of several tissues and organs, the production of hormones and a plenty of sensing signalling and regulatory molecules. Our body are replacing billions of cells every day and the food is the source for their renewal.

The diets of organisms could also affect the composition of their genes. Since organisms construct their DNA using building blocks they get from food, the composition of food could alter an organism's DNA. Recent experimental findings revealed a previously hidden relationship between cellular metabolism and evolution, and provided new insights into how DNA sequences can be influenced by adaptation to different diets [1]. Eating well is part of the strategy that can reduce our risk of any chronic disease and even improve the condition of our genes.

There is not "one rule fits all" and the commonly accepted recommendations such as low sugar, low salt, and a good variety of nutrients might be too simplistic considering also that the food choices could be influenced by genetics. The perception of taste varies according to the genetic makeup and not only taste but also the general eating behaviour of humans including the 24-h calorie intake, the meal size are controlled by our genes. Studies on offspring of obese families and monogynous twins have found links between genetic makeup and nutrient selection. The ability to sense and respond to fluctuations in environmental nutrient levels is a requisite for life. Different pathways that detect intracellular and extracellular levels of sugars, amino acids and lipids, and their end products, are integrated at higher hierarchical level via hormonal and nervous signals. The maintenance of a stable energy balance is a finely tuned homeostatic mechanisms able to store or mobilize the energy fuels stored in different tissues. Nutrient sensing pathways are commonly deregulated in human metabolic diseases [2]. What we eat and how much we eat is critical, but how we process it is perhaps even more important. Through thousands of little sensors, the gut has the immense task of managing all the 
information contained in the food we intake and the interactions and products derived from the huge population of microorganisms present in the gastrointestinal tract which we call collectively the microbiome. Nutrient scarcity has operated as a strong pressure for selecting efficient mechanisms of nutrient sensing in all organisms. Considering the importance of nutrient homeostasis for all living organisms, and for human health in particular, it is surprising that we know relatively little about direct nutrient sensing mechanisms. Most cells in multicellular eukaryotes are not directly exposed to changes in environmental nutrients, and homeostatic responses aimed to maintain circulating nutrient levels within a narrow range exist also in mammals mostly using complex signalling system as hormones, which act as long-range signals to facilitate the coordination of coherent responses in the whole organism. Understanding normal nutrient sensing mechanisms is a prerequisite for designing better interventions against human disease beyond obesity and type 2 diabetes.

Feeding is essential. So much so that according to the World Health Organization, adequate nutrition and health are to be considered fundamental human rights, closely related to each other. The health of the populations, both rich and poor, of the planet is strongly influenced by the level and quality of nutrition. A correct diet is a valuable tool for prevention for many diseases, and for management and treatment in many others. According to the WHO, for example, almost three million lives could be saved every year in the world thanks to sufficient consumption of fresh fruit and vegetables. The proportion of the types of foods and the quality of the foods we eat are the basis of complete human development, both physical and mental. On the other hand, poor quality food, contaminated or not properly stored can constitute substantial risk factors and are the cause of disease and death for millions of people every year. Furthermore, even an unbalanced or incorrect diet can generate disorderly conditions or real pathologies that are, in many cases, even fatal. Proper nutrition is, together with adequate physical activity and the control of other risk factors, such as smoking or alcohol, a fundamental element in the prevention of numerous pathological conditions. In fact, it has now been shown that lifestyle and food choices act incisively in the possibility of avoiding the development of diseases, controlling their evolution or, on the contrary, provoking their onset. In recent years, people worldwide have developed an increased popularity for weight loss program, diet plans and weight maintenance programs with little research done on the effectiveness of those programs. Meanwhile, obesity has been increasing in prevalence due to many social determinants such as easy access to various fast foods, and lack of physical activity. In 2016, World Health Organization (WHO) reported that more than 1.9 billion people in the world were overweight and over 650 million people were obese which has tripled in number since 1975. Also, obesity is a known risk factor for many metabolic disorders like type 2 diabetes, coronary heart disease, malignancies, osteoarthritis and respiratory disorders. Weight loss usually leads to the improvement in the overall wellbeing of patients and their metabolic and cardiovascular biomarkers such as systolic and diastolic blood pressure, blood glucose, insulin, total serum cholesterol, inflammatory biomarkers and low-density lipoprotein.

There are many forms of diet and exercise programs available for weight loss, however, one of the recently recognized diet changes is the alternate day fasting which includes eating $20 \%$ of energy requirements on a fast day and then consume food ad libitum on the feed days which has been suggestive to be highly effective for weight loss [3]. All the peoples of the world, all the great spiritual traditions, have promoted fasting practices, for religious, devotional, and therapeutic reasons. Fasting is often associated with Lent and with the Nativity fast or the season of Ramadan for Muslims. Islamic Ramadan is a 28-30 day fast in which food and drink are prohibited during the daylight hours. Greek Orthodox Christians fast for a total of 180-200 days each year, and their main fasting periods are the Nativity Fast (40 days prior to Christmas), Lent (48 days prior to Easter), and the Assumption (15 days in August). Many other cultures and religions around the world fast throughout the year. While the duration, practice, and specific reasons differ, all fasts have the similar goals of showing sacrifice and cleansing oneself. Religions and philosophies that practice fasting include: Buddhism, Christianity, Islam, Judaism, Taoism, Jainism, and Hinduism. Fasting has been around for centuries and will continue to be a regular. Some of the more favourable effects of these fasts include the lowering of body mass, total cholesterol, LDL-C, and the LDL-C/ HDL-C ratio. The Biblical-based Daniel Fast prohibits the consumption of animal products, refined carbohydrates, food additives, preservatives, sweeteners, flavourings, caffeine, and alcohol. It is most commonly partaken for 21 days, although fasts of 10 and 40 days have been observed [4, 5].

As a species, we have metabolic flexibility with the capability to rely on alternative fuel sources for energy. Humans are not dependent on exogenous sources of glucose for optimal function; rather, we have evolved over millennia to adjust to changing conditions and adapt to both scarcity and abundance. As Sir Randle explained in the Lancet in 1963, "Substrate metabolism in the normal human body is flexible. Our bodies have evolved to utilize different fuel sources depending on their availability." Moderate ketosis usually appears after a fast of a few hours, Ketone bodies are uel substrates, which uniquely can substitute and alternate with glucose under conditions of food deficiency, to act to preserve muscle protein during systemic inflammation and as an important component of the metabolic defence against insulin induced hypoglycaemia, and to increase myocardial blood flow and cardiac output in patients with heart failure. On this pathophysiological background the ketogenic diet became 
popular as a therapy for epilepsy. This diet has been more recently adopted as an effective strategy to induce a remarkable weight loss in people with obesity besides its well-known action on the brain metabolism and neuronal function and protection from epilepsy.

Intermittent fasting and chronic caloric restriction have been seen as low-protein high-carbohydrate diets may be the key to longevity, and healthy brain ageing in particular, according to Devin Wahl of the University of Sydney, who shows that unrestricted low-protein, high-carbohydrate diets have similar protective benefits for the brain as calorie restriction, which is well known for its longevity benefits although not sustainable in humans [6].

Low carbohydrate diets, which restrict carbohydrate in favour of increased protein or fat intake, or both, are a popular weight-loss strategy. However, the long-term effect of carbohydrate restriction on mortality is controversial and could depend on whether dietary carbohydrate is replaced by plantbased or animal-based fat and protein. In the ARIC cohort, a huge longitudinal study, there was a U-shaped association between the percentage of energy consumed from carbohydrate and mortality: a percentage of 50-55\% energy from carbohydrate was associated with the lowest risk of mortality. Low carbohydrate dietary patterns favouring animal-derived protein and fat sources, from sources such as lamb, beef, pork, and chicken, were associated with higher mortality, whereas those that favoured plant-derived protein and fat intake, from sources such as vegetables, nuts, peanut butter, and wholegrain breads, were associated with lower mortality, suggesting that the source of food notably modifies the association between carbohydrate intake and mortality [7].

In this special issue, the beneficial effects of the Mediterranean Diet are also illustrated. In recent years, extensive and significant research has been published that gives important signs of the existence of a causal relationship between the Mediterranean diet and the reduction in the incidence of cardiovascular diseases, tumors. and other important diseases. The first observational study on the Mediterranean Diet, which became famous as the "Seven Countries Study", was conducted by the American biologist and physiologist Ancel Keys in which the diets adopted by the United States, Italy, Finland, Greece, Yugoslavia, the Netherlands and Japan were compared to verify their benefits and critical points in terms of cardiovascular health. and today - only 50 years from the "discovery" of this characteristic, traditional food model the UNESCO declares that this century-long cookery culture, belonging to populations overlooking the Mediterranean Basin, represents a "Cultural Heritage of Humanity".

Lifestyle and individual and social behaviours influence people's nutritional choices and health. The relationship with food is deeply determined by a complex network of different factors, including the relationship with one's body, the reaction to traumatic events, emotional and family relationships, and many others. Several different socio-economic factors have caused a large portion of the population to adopt unhealthy eating habits that can undermine healthcare systems, unless current trends are inverted towards more sustainable lifestyle models. Even though a dietary plan inspired by the principles of the Mediterranean Diet is associated with numerous health benefits and has been demonstrated to exert a preventive effect towards numerous pathologies, including obesity, its use is decreasing and it is now being supplanted by different nutritional models that are often generated by cultural and social changes. Directing governments' political actions towards spreading adherence to the Mediterranean Diet's principles as much as possible among the population could help to tackle the obesity epidemic, especially in childhood.

Probably long before Hippocrates nutrition has always been seen with an ambivalent meaning or indispensable means for life, but also as an element that could induce disease and therefore it has already been thought to propose dietary plans or a selection of foods or one lifestyle that could also be a therapy for diseases usually produced by nutrition. Plato enemy of any excess said that diets did not serve to prolong life indefinitely but to be happy and in possession of the right psychophysical balance, which did not depend on an obsessive diet but on the result of a virtuous circle. "Know yourself is the motto of Socrates, understood as the ability to read and listen to one's own body and consequently to establish what is good and what is bad for everyone's health, because it is precisely the measure and the rule that make men.

This special issue intends to reiterate the importance of nutrition as a cause of the most important chronic diseases and the diet approach as a fundamental therapeutic strategy and to underlines how much difficult is to tailor a diet for an individual phenotype and even more difficult acting in different directions and with all the means we can adopt to stop the spread of obesity and its complications.

It is important to proceed with a critical review of the current nutritional approach and the diet models which are currently put forward, bearing in mind the importance of the scientific evidence that led to the consideration that only for the Mediterranean Diet strong evidences have been provided which seem to be effective in different pathological situations. We must support this diet not just as a food model, but also as the most appropriate regime for disease prevention, a sort of comprehensive and integrated lifestyle plan from a sustainable food system, and as a consequence, to support and speed up food system transformation as suggested by the EAT-Lancet Commission on Healthy Diets for Sustainable Food Systems [8] which links nutritional targets with environmental sustainability considering the triple challenges of obesity, undernutrition, and climate change, which interact and affect human and planetary health. In the age of precision and personalized medicine it is possible to believe, however, that the time has come to think of a dietary approach consistent with our 
general knowledge and with an ever deeper knowledge of the genotypic and phenotypic characteristics of each individual to whom we must prescribe a diet.

The Authors of the articles collected in the present special issue provide a comprehensive theoretical approach to the analysis of nutrition related diseases and how to treat these diseases with classic or innovative diet approaches. They help us to understand the physiological mechanisms that determine the nutritional interactions between humans with its environment, and the consequences of these interactions in terms of health, immune responses, and lifespan moreover it can also help to address the epidemic of obesity and related metabolic diseases.

\section{Compliance with ethical standards}

Conflict of interest The author declares that there is no conflict of interest regarding the publication of this article.

\section{References}

1. Seward EA, Kelly S. Dietary nitrogen alters codon bias and genome composition in parasitic microorganisms. Genome Biol. 2016;17: 226.
2. Efeyan A, Comb WC, Sabatini DM. Nutrient-sensing mechanisms and pathways. Nature. 2015;517(7534):302-10.

3. de Cabo R, Mattson MP. Effects of intermittent fasting on health, aging, and disease. N Engl J Med. 2019;381:2541-255.

4. Trepanowski JF, Bloomer RJ. The impact of religious fasting on human health. Nutr J. 2010;9:57.

5. Persynaki A, Karras S, Pichard C. Unraveling the metabolic health benefits of fasting related to religious beliefs: A narrative review. Nutrition. 2017;35:14-20.

6. Devin Wahl, Samantha M. Solon-Biet,Qiao-Ping Wang, Jibran A. Wali,Tamara Pulpitel, Ximonie Clark, David Raubenheimer, Alistair M. Senior, David A. Sinclair, Gregory J. Cooney, Rafael de Cabo, Victoria C. Cogger, Stephen J. Simpson, and David G. Le Couteur. Comparing the effects of low-Proteinand high-carbohydrate Dietsand caloric restriction on brain aging in mice cell reports 2018;25:2234-2243.

7. Seidelmann SB, Claggett B, Cheng S, Henglin M, Shah A, Steffen LM, et al. Articles Dietary carbohydrate intake and mortality: a prospective cohort study and meta-analysis. Lancet Public Health. 2018;3:e419-28.

8. Willett, et al. Food in the Anthropocene: the EAT-Lancet Commission on healthy diets from sustainable food systems. Walter The Lancet Commissions. 2019;393(10170):447-92.

Publisher's note Springer Nature remains neutral with regard to jurisdictional claims in published maps and institutional affiliations. 